Originalien

Chirurg 2021 · 92:830-837

https://doi.org/10.1007/s00104-020-01303-7

Online publiziert: 23. Oktober 2020

(C) Der/die Autor(en) 2020

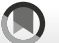

Check for
updates

Y. Carmen Ahmadzadeh' ${ }^{1}$ Th. Schmitz-Rixen ' - D. Böckler ${ }^{2}$ R. T. Grundmann ${ }^{3}$

${ }^{1}$ Klinik für Gefäß- und Endovascularchirurgie und des Universitären Wundzentrums, Klinikum der GoetheUniversität, Frankfurt/M, Deutschland

${ }^{2}$ Klinik für Gefäßchirurgie und Endovaskuläre Chirurgie, Universitätsklinikum Heidelberg, Heidelberg, Deutschland

${ }^{3}$ Deutsches Institut für Gefäßmedizinische Gesundheitsforschung (DIGG) der Deutschen Gesellschaft für Gefäßchirurgie und Gefäßmedizin, Berlin, Deutschland

\title{
Fallzahlaufkommen und Qualitätsindikatoren bei der Versorgung des abdominellen Bauchaortenaneurysmas
}

pagiert worden. Die vorliegende Untersuchung stellte sich deshalb die Frage, ob der MTL30 auch als Qualitätssurrogatparameter im Bauchaortenaneurysma(AAA)-Register des Deutschen Instituts für Gefäßmedizinische Gesundheitsforschung (DIGG) der Deutschen Gesellschaft für Gefäßchirurgie und Gefäßmedizin (DGG) zur Anwendung kommen könnte. Dies zum einen unter der Vorstellung, dass Klinikletalität und Komplikationsraten - zumindest bei offener Versorgung (OAR) des AAA - nicht unbeträchtlich sind und dass zum anderen die Ergebnisse auch von der Klinikstruktur bestimmt werden. Zwar können keine definitiven Mindestmengen gefordert werden, jedoch besteht eine signifikante inverse Beziehung zwischen Krankenhausfallaufkommen und Klinikletalität bei Versorgung des intakten AAA (iAAA), die allerdings bei OAR sehr viel ausgeprägter als bei endovaskulärer Versorgung (EVAR) des Aneurysmas ist (Übersicht in [5]). Wir haben deshalb in der vorliegenden Analyse die Registerergebnisse der letzten Jahre hinsichtlich des MTL30 überprüft und dabei untersucht, ob sich dieser Parameter zum Fallaufkommen einer Klinik korrelieren lässt.

\section{Material und Methodik}

Das multizentrische Patientenkollektiv dieser retrospektiven Datenauswertung entstammt dem AAA-Register des DIGG der DGG der Jahre 2013 bis 2017. Alle teilnehmenden Kliniken haben in diesem Register ihre Patienten auf freiwilliger Basis prospektiv dokumentiert. Die Angaben zu Patienten und ihren Klinikaufenthalten wurden pseudonymisiert in das AAA-Register eingetragen. Betrachtet wurden in der vorliegenden Arbeit alle Patienten mit elektiver Versorgung eines nichtrupturierten, symptomatischen oder asymptomatischen Bauchaortenaneurysmas, bei denen die Angaben zu Aneurysmadurchmesser, Klinikletalität, Liegedauer und Transfer in ein anderes Krankenhaus vorhanden waren. Weiterhin wurden nur Kliniken bei der Analyse berücksichtigt, die mindestens zwei EVAR bzw. zwei OAR jährlich dokumentiert haben. Klinische Angaben zu dem Patientenkollektiv finden sich in $\bullet$ Tab. 1. Wie ersichtlich, wurden wesentlich mehr Patienten mit EVAR $(n=14.282)$ als mit OAR $(n=3923)$ versorgt und es führten auch weniger Kliniken OAR $(n=158)$ als EVAR $(n=212)$ durch. Allerdings beteiligten sich an dem Register bei EVAR nur 31 Kliniken über die gesamte Dauer von 5 Jahren, bei OAR waren es 16 Kliniken.

Es wurde für EVAR und OAR separat überprüft, inwieweit die perioperativen Ergebnisse vom jährlichen Fallaufkommen der teilnehmenden Kliniken abhängig waren. Hierzu wurden die Kliniken

Im Auftrag des DIGG gGmbH 


\begin{tabular}{|c|c|c|c|}
\hline Parameter & EVAR & OAR & $\boldsymbol{P}$ \\
\hline Patienten, $n$ & 14.282 & 3923 & - \\
\hline Teilnehmende Kliniken, $n$ & 212 & 158 & - \\
\hline Männer, $n$ (\%) & $12.382(86,7)$ & $3275(83,5)$ & 0,000 \\
\hline Frauen, $n(\%)$ & $1900(13,3)$ & $648(16,5)$ & 0,000 \\
\hline Patienten $\geq 80$ Jahre, $n(\%)$ & $3194(22,4)$ & $360(9,2)$ & 0,000 \\
\hline Patienten $<80$ Jahre, $n(\%)$ & $11.088(77,6)$ & $3562(90,8)$ & 0,000 \\
\hline Alter insgesamt, in Jahren $\mathrm{MW} \pm \mathrm{SD}$, Median & $73,00 \pm 8,43,74$ & $69,08 \pm 8,54,70$ & 0,000 \\
\hline Alter der Männer, in Jahren $\mathrm{MW} \pm \mathrm{SD}$, Median & $72,67 \pm 8,40,74$ & $70,49 \pm 8,79,70$ & 0,000 \\
\hline Alter der Frauen, in Jahren $M W \pm S D$, Median & $75,19 \pm 8,35,76$ & $68,80 \pm 9,48,72$ & 0,000 \\
\hline $\begin{array}{l}\text { AAA-Durchmesser Männer, in mm MW } \pm \text { SD, } \\
\text { Median }\end{array}$ & $54,95 \pm 11,22,54$ & $57,26 \pm 13,41,55$ & 0,000 \\
\hline $\begin{array}{l}\text { AAA-Durchmesser Frauen, in } \mathrm{mm} M W \pm S D \text {, } \\
\text { Median }\end{array}$ & $51,59 \pm 11,70,52$ & $54,39 \pm 12,29,53$ & 0,000 \\
\hline $\begin{array}{l}\text { Durchschnittliche Liegedauer, in Tagen } \\
M W \pm S D\end{array}$ & $9,89 \pm 15,63$ & $17,49 \pm 24,30$ & 0,000 \\
\hline$A S A \geq 3, n(\%)$ & $10.396(72,8 \%)$ & $2854(72,8 \%)$ & 0,960 \\
\hline Z. n. Myokardinfarkt, $n(\%)$ & $2372(16,6 \%)$ & $637(16,2 \%)$ & 0,580 \\
\hline Renale Begleiterkrankung, $n(\%)^{\text {a }}$ & $3096(21,7 \%)$ & $916(23,3 \%)$ & 0,025 \\
\hline Z.n. Apoplex, $n$ (\%) & $1382(9,7 \%)$ & $355(9,0 \%)$ & 0,236 \\
\hline \multicolumn{4}{|c|}{$\begin{array}{l}\text { EVAR endovaskuläre Aneurysmaversorgung, OAR offene Aneurysmaversorgung } \\
\text { "Unter "renale Begleiterkrankung“ wurden alle Patienten erfasst mit Z. n. Nieren Tx,Z. n. Nephrektomie } \\
\text { und solche, die eine eingeschränkte Nierenfunktion mit einer GFR <90 ml/min (ab Stadium 2) } \\
\text { aufwiesen }\end{array}$} \\
\hline
\end{tabular}

Tab. 2 Perioperatives Outcome bei EVAR und OAR

\begin{tabular}{|l|l|l|l}
\hline Parameter & $\begin{array}{l}\text { EVAR } \\
(\boldsymbol{n}=\mathbf{1 4 . 2 8 2})\end{array}$ & $\begin{array}{l}\text { OAR } \\
(\boldsymbol{n}=\mathbf{3 9 2 3})\end{array}$ & $\boldsymbol{p}$ \\
\hline MTL30-Fälle, $n(\%)$ & $719(5,0)$ & $566(14,4)$ & 0,000 \\
\hline Sterbefälle, $n(\%)$ & $180(1,3)$ & $191(4,9)$ & 0,000 \\
\hline Fälle mit Transfer in eine andere Klinik, $n(\%)$ & $207(1,4)$ & $102(2,6)$ & 0,000 \\
\hline Fälle mit Liegedauer über $\geq 30$ Tage, $n(\%)$ & $384(2,7)$ & $328(8,4)$ & 0,000 \\
\hline EVAR endovaskuläre Aneurysmaversorgung, OAR offene Aneurysmaversorgung & \\
\hline
\end{tabular}

bei EVAR von Nr. 1 bis 212 und bei OAR von Nr. 1 bis 158 entsprechend dem ansteigenden jährlichen Fallaufkommen gereiht und dann in Quintile unterteilt, mit Klinikquintile 1 mit dem geringsten und Klinikquintile 5 mit dem höchsten Fallaufkommen. Die Mittelwerte der Querschnittsdaten einer Klinik wurden individuell erstellt, in dem für jede Klinik ihr jährliches Fallaufkommen berechnet wurde. Eine Klinik kann damit nur einem Klinikquintil zugehören.

\section{Statistik}

Die Analyse erfolgte primär mit dem Tabellenkalkulationsprogramm Excel für Windows (Microsoft Excel 2019 für
Zusammenhang interpretiert, eine Signifikanz von $p<0,05$ vorausgesetzt.

\section{Ergebnisse}

\section{Gesamtkrankengut}

Klinikletalität, Fälle mit MTL30, Fälle mit Liegedauer $\geq 30$ Tage und Fälle mit Transfer in ein anderes Krankenhaus sind vergleichend für EVAR und OAR in • Tab. 2 wiedergegeben. Die Ergebnisse waren für alle Parameter nach EVAR signifikant besser als nach OAR, mit einer Klinikletalität von $1,3 \%$ vs. $4,9 \%$ und einem MTL30 von $5,0 \%$ vs. $14,4 \%$.

\section{Abhängigkeit der Ergebnisse vom Fallaufkommen}

Für EVAR ließ sich keine Beziehung zwischen Fallaufkommen und Ergebnis nachweisen, das galt für Klinikletalität (Quintile 1: 1,0\%; Quintile 5: 1,3\%) und MTL30 (Quintile 1: 5,3\%; Quintile 5: 5,3\%) gleichermaßen (• Tab. 3). Auch bei OAR bestand keine signifikante Beziehung zwischen Fallaufkommen und Outcome: Klinikletalität Quintile 1: 5,8\%, Quintile 5: 3,5\%; $p=0,505$; MTL30 Quintile 1: 16,4\%, Quintile 5: 12,2\%, $p=0,110$ (•Tab. 4).

\section{Kliniken mit $>30$ vs. <30 Fälle/Jahr (EVAR + OAR zusammengefasst)}

Neunundsiebzig Kliniken (36,6\%) mit insgesamt 10.297 Patienten gaben an, pro Jahr $\geq 30$ Fälle (OAR und EVAR zusammengefasst) $\mathrm{zu}$ behandeln (im Mittel 47,9 Fälle/Jahr). Ihnen stehen $137(63,4 \%)$ Kliniken mit insgesamt 7908 Patienten gegenüber, die pro Jahr weniger als 30 Fälle (OAR und EVAR zusammengefasst) behandelten (im Mittel 17,5 Fälle/Jahr). Die Ergebnisse ( $\geq 30$ vs. $<30$ Fälle/Jahr) waren nicht signifikant unterschiedlich: Klinikletalität $1,9 \%$ vs. 2,2\%; MTL30 6,9\% vs. 7,3\%; Liegezeit über 30 Tage $3,7 \%$ vs. 4,2\%; Fälle mit Transfer $2,0 \%$ vs. $1,3 \%$. 
Chirurg 2021 · 92:830-837 https://doi.org/10.1007/s00104-020-01303-7

(c) Der/die Autor(en) 2020

\section{Y. C. Ahmadzadeh • T. Schmitz-Rixen • D. Böckler · R. T. Grundmann}

\section{Fallzahlaufkommen und Qualitätsindikatoren bei der Versorgung des abdominellen Bauchaortenaneurysmas}

\section{Zusammenfassung}

Hintergrund. Der MTL30 (Mortalität, Transfer, Liegezeit) wurde als Surrogatparameter zur Evaluation der Qualität potenziell komplikationsträchtiger viszeralchirurgischer Eingriffe vorgeschlagen.

Zielsetzung. Es wurde überprüft, inwieweit sich der MTL30 zu den Ergebnissen des Bauchaortenaneurysma(AAA)-Registers des Deutschen Instituts für Gefäßmedizinische Gesundheitsforschung (DIGG) der Deutschen Gesellschaft für Gefäßchirurgie und Gefäßmedizin (DGG) und zum Fallaufkommen der Kliniken korrelieren lässt.

Material und Methoden. Insgesamt 14.282 Patienten wurden endovaskulär (EVAR) und 3923 Patienten offen (OAR) elektiv wegen eines AAA versorgt. Bestimmt wurden Fallaufkommen der behandelnden Kliniken,
Klinikletalität, Liegezeit und Verlegung in ein anderes Akutkrankenhaus 30 Tage nach dem Indexeingriff.

Ergebnisse. Die Klinikletalität machte bei EVAR $1,3 \%$, bei OAR $4,9 \%$ aus $(p=0,000)$, der MTL30 5,0\% vs. 14,4\% ( $p=0,000)$. Für EVAR ließ sich keine Beziehung zwischen Fallaufkommen und Klinikletalität (Quintile 1: 1,0\%; Quintile 5: 1,3\%) sowie Fallaufkommen und MTL30 (Quintile 1: 5,3\%; Quintile 5: $5,3 \%$ ) nachweisen. Auch bei OAR bestand keine signifikante Beziehung zwischen Fallaufkommen und Klinikletalität (Quintile 1: 5,8\%, Quintile 5: 3,5\%; $p=0,505)$ und Fallaufkommen und MTL30 (Quintile 1: $16,4 \%$, Quintile 5: 12,2\%, $p=0,110)$. Bei einer Klinikletalität von 7,2 (5-10) \% betrug der MTL30 bei OAR 17,6\%. Sowohl bei EVAR als auch bei OAR korrelierte die stationäre Aufenthaltsdauer signifikant mit Klinikletalität und MTL30.

Diskussion. Eine eindeutige Beziehung zwischen Krankenhausfallaufkommen und Klinikletalität ließ sich im AAA-Register des DIGG nicht aufzeigen. Das gleiche galt für den MTL30. Ob demnach der MTL30 gegenüber der Erfassung von Klinikletalität und stationärer Liegezeit als Qualitätsparameter einen Zusatznutzen bietet, muss offenbleiben.

Schlüsselwörter

Bauchaortenaneurysma · Endovaskuläre Versorgung · Offene Versorgung · Krankenhausfallaufkommen· Qualitätsindikatoren

\section{Case load and quality indicators in the treatment of abdominal aortic aneurysms}

\section{Abstract}

Background. The MTL30 (mortality, transfer, length of stay) was proposed as a surrogate parameter for evaluating the quality of large and potentially complication-prone visceral surgical interventions.

Objective. The aim of this study was to find out to what extent the MTL30 can be correlated with the results of the abdominal aortic aneurysm (AAA) registry of the German Institute for Vascular Health Research (DIGG) of the German Society for Vascular Surgery and Vascular Medicine (DGG) and with the case volume of the participating hospitals. Material and methods. Elective endovascular abdominal aortic aneurysm repair (EVAR) was performed in 14,282 patients and open repair (OAR) in 3923 patients. Case volume of the treating hospitals, hospital mortality, length of stay and transfer to another acute care hospital were determined 30 days after the index intervention.

Results. The hospital mortality was $1.3 \%$ for EVAR and $4.9 \%$ for OAR $(p=0.000)$, the MTL30 was $5.0 \%$ and $14.4 \%$, respectively $(p=0.000)$. For EVAR, no relationship between case volume and hospital mortality (quintile 1: $1.0 \%$; quintile $5: 1.3 \%$ ) as well as case volume and MTL30 (quintile 1: 5.3\%; quintile 5:5.3\%) could be demonstrated. Also in OAR there was no significant relationship between case volume and hospital mortality (quintile 1 : $5.8 \%$, quintile $5: 3.5 \% ; p=0.505$ ) and case volume and MTL30 (quintile 1: 16.4\%, quintile $5: 12.2 \%, p=0.110$ ). With a hospital mortality rate of $7.2 \%(5-10 \%)$ the MTL30 for OAR was $17.6 \%$. In both EVAR and OAR, the length of stay correlated significantly with hospital mortality and MTL30.

Discussion. A clear relationship between hospital case volume and hospital mortality could not be shown in the AAA registry of the DIGG. The same was true for the MTL30. It remains to be seen whether the MTL30 offers an additional benefit compared to the recording of hospital mortality and inpatient length of stay as a quality parameter.

\section{Keywords}

Abdominal aortic aneurysm - Endovascular repair - Open repair - Hospital case volume . Quality indicators
Kliniken mit $<20$ vs. $>20$ Fälle/Jahr (EVAR + OAR zusammengefasst)

Einhundertdreiunddreißig Kliniken (61,6\%) mit insgesamt 14.735 Patienten gaben an, pro Jahr $\geq 20$ Fälle (OAR und EVAR zusammengefasst) zu behandeln (im Mittel 39,2 Fälle/Jahr). Ihnen stehen 83 (38,4\%) Kliniken mit insgesamt 3470 Patienten gegenüber, die pro Jahr weniger als 20 Fälle (OAR und EVAR zusammengefasst) behandelten (im Mittel
13,5 Fälle/Jahr). Die Ergebnisse ( $\geq 20$ vs. $<20$ Fälle/Jahr) waren nicht signifikant unterschiedlich: Klinikletalität 2,0\% vs. 2,0\%; MTL30 7,0\% vs. 7,2\%; Liegezeit über 30 Tage $3,8 \%$ vs. $4,3 \%$; Fälle mit Transfer $1,8 \%$ vs. $1,3 \%$.

\section{Kliniken mit Klinikletalität 5-10\% bei OAR}

Dreiunddreißig von 158 Kliniken (20,9\%) mit insgesamt 330 Fällen/Jahr (medianes jährliches Fallvolumen pro Klinik $n=7$ ) wiesen bei OAR eine Klinikletalität von $5,0-10,0 \%$ auf. Die Klinikletalität betrug in diesem Kollektiv über alle 7,2\%, der MTL30 17,6\%, Fälle mit Liegezeit $\geq 30$ Tage $10,2 \%$ und Transfer ebenfalls $10,2 \%$. 


\begin{tabular}{|c|c|c|c|c|c|c|}
\hline Klinikquintile & $\begin{array}{l}\text { Anzahl Klini- } \\
\text { ken }\end{array}$ & $\begin{array}{l}\text { Fallzahl/Jahr, } n \text { (Me- } \\
\text { dian) }\end{array}$ & $\begin{array}{l}\text { Sterbefälle, } n \\
\text { (\%) }\end{array}$ & $\begin{array}{l}\text { Fälle MTL30, } n \\
\text { (\%) }\end{array}$ & $\begin{array}{l}\text { Fälle Liegedauer } \geq 30 \text { Ta- } \\
\text { ge, } n(\%)\end{array}$ & $\begin{array}{l}\text { Fälle Transfer, } n \\
\text { (\%) }\end{array}$ \\
\hline $\mathrm{Q} 1, n=1071$ & 44 & $2-11,5(8)$ & $11(1,0)$ & $57(5,3)$ & $42(3,9)$ & $9(0,8)$ \\
\hline$Q 2, n=2012$ & 43 & $11,8-16,0(13,8)$ & $21(1,0)$ & $98(4,9)$ & $58(2,9)$ & $25(1,2)$ \\
\hline $\mathrm{Q} 3, n=2609$ & 43 & $16,3-22,8(19,2)$ & $33(1,3)$ & $127(4,9)$ & $66(2,5)$ & $38(1,5)$ \\
\hline Q 4, $n=3349$ & 42 & $23-32,0(26,1)$ & $46(1,4)$ & $159(4,8)$ & $85(2,5)$ & $35(1,0)$ \\
\hline Q 5, $n=5241$ & 40 & $32,5-114(43)$ & $69(1,3)$ & $278(5,3)$ & $133(2,5)$ & $100(1,9)$ \\
\hline
\end{tabular}

\begin{tabular}{|c|c|c|c|c|c|c|}
\hline Klinikquintile & $\begin{array}{l}\text { Anzahl Klini- } \\
\text { ken }\end{array}$ & $\begin{array}{l}\text { Fallzahl/Jahr, } n \text { (Me- } \\
\text { dian) }\end{array}$ & $\begin{array}{l}\text { Sterbefälle, } n \\
(\%)\end{array}$ & $\begin{array}{l}\text { Fälle MTL30, } n \\
\text { (\%) }\end{array}$ & $\begin{array}{l}\text { Fälle Liegedauer } \geq 30 \text { Ta- } \\
\text { ge, } n \text { (\%) }\end{array}$ & $\begin{array}{l}\text { Fälle Transfer, } n \\
\text { (\%) }\end{array}$ \\
\hline Q 1, n= 208 & 31 & $2-3(2,6)$ & $12(5,8)$ & $34(16,4)$ & $21(10,1)$ & $2(1,0)$ \\
\hline Q 2,n=464 & 31 & $3,2-5,2(4,0)$ & $24(5,2)$ & $78(16,8)$ & $48(10,3)$ & $13(2,8)$ \\
\hline $\mathrm{Q} 3, n=630$ & 30 & $5,3-6,8(6,1)$ & $49(7,8)$ & $104(16,5)$ & $46(7,3)$ & $13(2,0)$ \\
\hline $\mathrm{Q} 4, n=830$ & 33 & $7-10(8,5)$ & $44(5,3)$ & $132(15,9)$ & $79(9,5)$ & $20(2,4)$ \\
\hline Q 5, $n=1791$ & 33 & $10,8-53(17,5)$ & $62(3,5)$ & $218(12,2)$ & $134(7,5)$ & $54(3,0)$ \\
\hline
\end{tabular}

\section{Liegezeit und Klinikletalität}

\section{EVAR}

Es gab eine signifikante Beziehung zwischen Dauer der Liegezeit und Klinikletalität sowie MTL30. Dieser Zusammenhang war für den MTL30 ausgeprägter $(\mathrm{r}=0,81)$ als für die Sterblichkeit $(r=0,463$; - Abb. 1). Des Weiteren wurde die stationäre Aufenthaltsdauer in Quintile unterteilt, nach Kliniken $(n=43)$ mit der längsten (Quintile 1) und Kliniken $(n=42)$ mit der kürzesten Liegezeit (Quintile 5). In Quintile 1 (2013 Patienten) betrug die Liegezeit im Mittel 15,1 \pm 35,6 (Median 9) Tage, die Klinikletalität war 1,7\%, der MTL30 $7,4 \%$. Die Vergleichszahlen in Quintile 5 (2845 Patienten) waren: Liegezeit im Mittel 7,2 $\pm 4,8$ (Median 6) Tage, Klinikletalität $1,0 \%$, MTL30 2,8\%. In Quintile 5 waren damit Klinikletalität $(p=0,042)$ und MTL30 $(p=0,000)$ signifikant günstiger als in Quintile 1.

\section{OAR}

Auch für OAR fand sich eine signifikante Beziehung zwischen Dauer der Liegezeit und Klinikletalität sowie MTL30, wiederum für den MTL30 ausgeprägter $(r=0,904)$ als für die Sterblichkeit $(r=0,552$; $\bullet$ Abb. 2). Die stationäre Aufenthaltsdauer, in Quintile unterteilt, ergab 32 Kliniken mit der längsten (Quin- tile 1) und ebenfalls 32 Kliniken mit der kürzesten Liegezeit (Quintile 5). In Quintile 1 (487 Patienten) betrug die Liegezeit im Mittel 30,1 $\pm 60,5$ (Median 14) Tage, die Klinikletalität war $6,8 \%$, der MTL30 26,3\%. Die Vergleichszahlen in Quintile 5 (693 Patienten) waren: Liegezeit im Mittel 12,2 $\pm 5,8$ (Median 11) Tage, Klinikletalität 2,2\%, MTL30 5,2\%. In Quintile 5 waren damit Klinikletalität $(p=0,000)$ und MTL30 $(p=0,000)$ signifikant günstiger als in Quintile 1.

\section{Diskussion}

In der vorliegenden Analyse machte die Klinikletalität bei elektiver endovaskulärer Versorgung des AAA 1,3\%, bei offener Versorgung 4,9\% aus. Die signifikant niedrigere Klinikletalität bei EVAR im Vergleich zu OAR war zu erwarten [1] - entsprechend auch dem Registerbericht für das Jahr 2018 [9] - und soll im Zusammenhang mit der Zielsetzung der Arbeit nicht weiter diskutiert werden. So nannten Bulder et al. [2] auf Basis von 51 Studien (189.022 Patienten) nach EVAR eine gepoolte 30-TageLetalität von $1,16 \%$ (95\%-Konfidenzintervall $0,92-1,39)$, verglichen mit $3,27 \%$ $(2,71-3,83)$ nach OAR $(p<0,001)$. Parallel zur Klinikletalität ergaben sich auch signifikante Unterschiede im MTL30, der bei EVAR 5,0\%, bei OAR 14,4\% ausmachte. Da Vergleichsdaten für ein gefäßchirurgisches Krankengut nicht zur Verfügung stehen, muss zur Bewertung des MTL30 auf die viszeralchirurgischen Analysen zurückgegriffen werden. Wiegering et al. [14] nannten für die Chirurgie des Rektumkarzinoms (StuDoQ | Rektumkarzinompopulation) bei 7142 Patienten einen MTL30 von $10,7 \%$, womit hier die offene Chirurgie des AAA mit einem MTL30 von $14,4 \%$ komplikationsträchtiger als die Chirurgie des Rektumkarzinoms wäre. Inwieweit ein alleiniger Vergleich der Klinikletalitäten zu einer ähnlichen Aussage gekommen wäre, muss offen bleiben, die Klinikletalität wurde von Wiegering et al. für das genannte Krankengut nicht aufgeführt. Die Autoren schlugen aber vor, bei Patienten mit radikaler Primärtumorresektion des Rektums einen MTL30-Wert $<20 \% \mathrm{zu}$ fordern. Weitere Angaben finden sich für die elektive Pankreatoduodenektomie, Wellner et al. [12] nannten als Qualitätsziele eine Krankenhaussterblichkeit von $\leq 7 \%$ und einen MTL30-Wert von $\leq 25 \%$.

Die Frage ist, welcher MTL30-Grenzwert als Qualitätsziel bei der Versorgung des AAA in unserem Register angegeben werden sollte. Hierzu wurden die Klinken zum einen einem Benchmarking hinsichtlich des Fallaufkommens unter- 

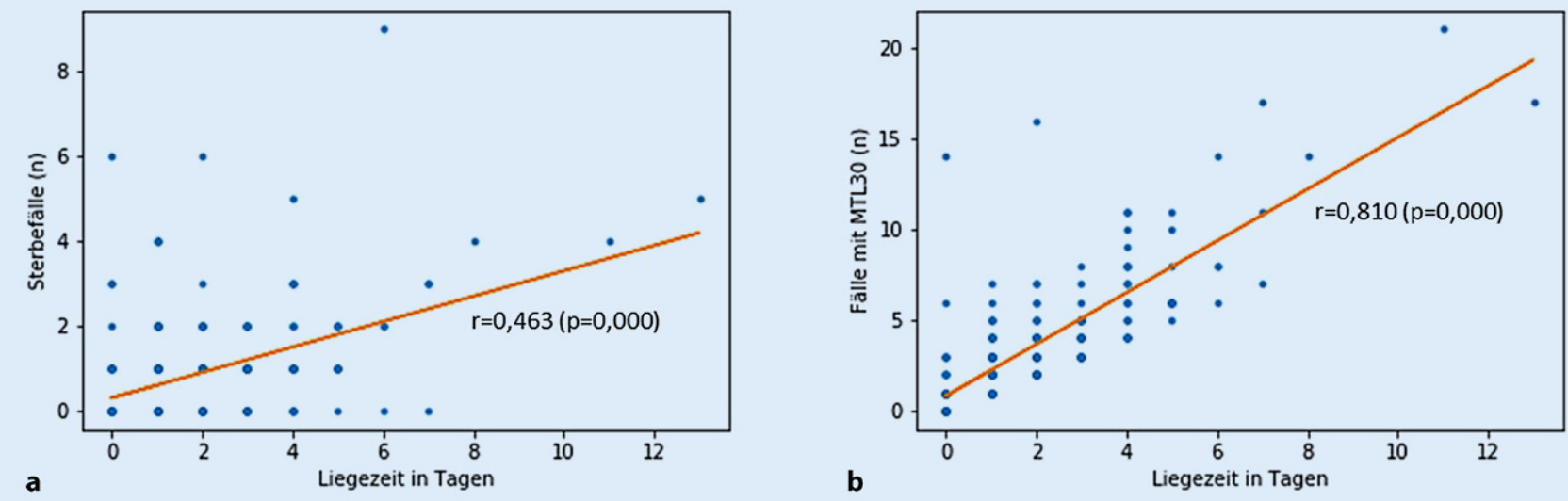

Abb. 1 A Korrelation von Klinikletalität (a) und MTL30 (b) zur stationären Aufenthaltsdauer bei EVAR.MTL Mortalität, Transfer, Liegezeit; EVAR endovaskuläre Aneurysmaversorgung
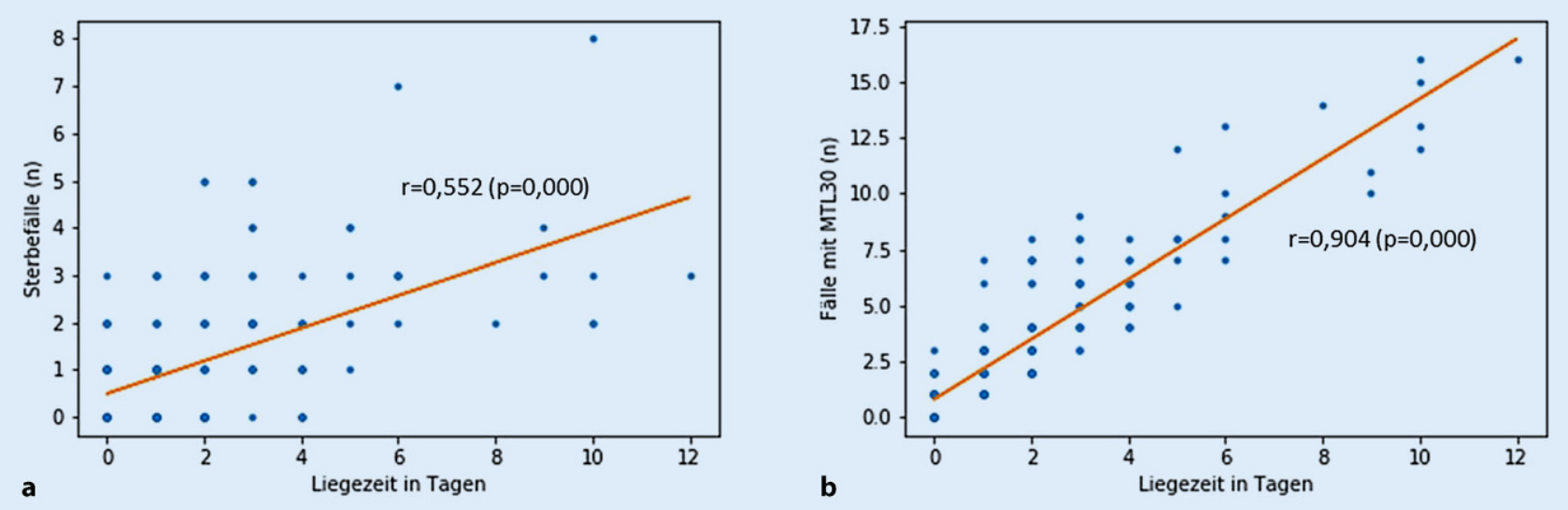

Abb. $2 \Delta$ Korrelation von Klinikletalität (a) und MTL30 (b) zur stationären Aufenthaltsdauer bei OAR. MTL Mortalität, Transfer, Liegezeit; OAR offene Aneurysmaversorgung

zogen und zum zweiten Qualitätsziele bzw. Fallvorgaben überprüft, die in den Leitlinien der European Society for Vascular Surgery (ESVS; [11]) und der Society for Vascular Surgery (SVS; [3]) für die elektive Versorgung des AAA gefordert werden.

Wie • Tab. 3 demonstriert, konnte für EVAR keine Beziehung zwischen Fallaufkommen einer Klinik und Klinikletalität oder MTL30 hergestellt werden, die Klinikquintile 1 mit im Median 8 EVAR/Jahr unterschied sich nicht von Klinikquintile 5 mit im Median 43 EVAR jährlich. Bei der niedrigen Klinikletalität und dem geringen MTL30-Wert (verglichen mit den Angaben zu den oben genannten komplexen viszeralchirurgischen Eingriffen) ist das Ergebnis nicht überraschend und entspricht den Literaturangaben. So ka- men Dua et al. [4] zu dem Schluss, dass bei Versorgung des AAA mit EVAR für eine Klinik lediglich 8 EVAR jährlich $\mathrm{zu}$ fordern seien, Zettervall et al. [16] schlugen $\geq 9$ EVAR jährlich als Mindestmenge vor. Nimptsch und Mansky [8] werteten deutsche DRG-Daten der Jahre 2009 bis 2014 aus. In dieser Untersuchung (41.678 Patienten mit EVAR) ließ sich zwischen Krankenhausfallaufkommen und Kliniksterblichkeit für EVAR ebenfalls keine signifikante Korrelation erstellen. Die Konsequenz ist, dass sich zum Klinikbenchmarking bei EVAR Angaben zu Fallzahlen und MTL30 nur eingeschränkt eignen. Eher reicht es aus, neben der Klinikletalität in einem Register die Krankenhausaufenthaltsdauer zu erfassen, wobei allerdings die in den USamerikanischen Registern angegebenen
Liegezeiten auf Deutschland nicht direkt übertragbar sind. In einer Erhebung der Vascular Quality Initiative (VQI) der SVS bezeichneten Zettervall et al. [17] bereits eine Aufenthaltsdauer von mehr als 2 Tagen bei 14.510 Patienten mit EVAR als „prolongiert", was in Abhängigkeit von der Komplikationsrate regional unterschiedlich in 16-43\% der Fall war. Sie sahen in der Bestimmung der Liegezeit einen Qualitätsparameter, da die Aufenthaltsdauer signifikant mit der Komplikationsrate korrelierte. Yin et al. [15] haben Daten der National Surgical Quality Improvement Program(NSQIP)-Datenbank der Jahre 2006 bis 2010 (11.539 Patienten mit EVAR) mit denen der Jahre 2011 bis 2015 (18.537 Patienten mit EVAR) miteinander verglichen. Sie berichteten für beide Beobachtungszeiträu- 
me eine gleichbleibend geringe 30-Tage-Letalität von $1,2 \%$. Bei abnehmender Majorkomplikationsrate von 5,1\% vs. $4,1 \%$ sank parallel die Krankenhausaufenthaltsdauer von 3,2 $\pm 5,3$ Tage auf $2,8 \pm 4,3$ Tage im nachfolgenden Zeit$\operatorname{raum}(p<0,001)$. Wir haben in der vorliegenden Untersuchung deshalb ebenfalls die Aufenthaltsdauer untersucht. Sie korrelierte signifikant mit Klinikletalität und MTL30 (• Abb. 1). In der Quintile mit der längsten Liegezeit waren Klinikletalität mit $1,7 \%$ vs. $1,0 \%$ und MTL30 mit $7,4 \%$ vs. $2,8 \%$ signifikant höher als in der Quintile mit der geringsten Liegezeit.

Da mittlerweile bei elektiver Versorgung des AAA generell aufgrund der niedrigeren Klinikletalität - wenn anatomisch/morphologisch möglich - EVAR der Vorzug vor OAR gegeben wird, wie auch hier in 78,5\% der Fälle, ist die ESVS bei Formulierung ihrer Leitlinie einen anderen Weg gegangen, um Mindestmengen zu deklarieren [11]. Sie hat Mindestmengen für den kombinierten Einsatz von OARplus EVAR definiert. Danach sollte die Versorgung eines AAA nur in Kliniken in Betracht gezogen werden, die wenigstens 30 Fälle jährlich versorgen, unabhängig davon, ob dies EVAR oder OAR ist, und sie sollte nicht in Kliniken mit weniger als 20 Fällen jährlich durchgeführt werden (OAR und EVAR zusammengefasst). Auf Registerauswertungen konnte sich die ESVS bei diesen Formulierungen nicht berufen, es handelte sich um Evidenzlevel-B- und -CAussagen. Wir haben hier erstmals in einem Register die Ansprüche der ESVS überprüft. Es zeigte sich, dass Kliniken, die EVAR und OAR zusammengefasst mehr als 30-mal pro Jahr durchführten, sich weder in Klinikletalität noch MTL30 von Kliniken unterschieden, die diesen Grenzwert unterschritten. Gleiches galt für die Überprüfung der „Sollnicht"-Empfehlung der ESVS: Kliniken, die pro Jahr weniger als 20 Fälle (OAR und EVAR zusammengefasst) behandelten, unterschieden sich nicht in Klinikletalität und MTL30 von Kliniken mit höherem Fallaufkommen. Diese Ergebnisse resultieren aus der Tatsache, dass die Mehrzahl der AAA in unserem Register mit EVAR versorgt wurde und die Klinikletalität bei EVAR damit im We- sentlichen die Gesamtletalität des Krankenguts bestimmte.

Auch bei OAR konnten wir im vorliegenden Kollektiv keine signifikante Beziehung zwischen Klinikletalität und MTL30 einerseits und Fallaufkommen andererseits demonstrieren (• Tab. 4). Dieses Ergebnis steht im Widerspruch $\mathrm{zu}$ anderen Untersuchungen [5] und beruht letztlich auf den geringen Fallzahlen, die uns gemeldet wurden. So kam selbst die höchste Klinikvolumenquintile auf nicht mehr als im Median 17,5 Fälle/Jahr.

Da wir auf Basis der Fallzahlen für den MTL30 bei OAR keinen eindeutigen Grenzwert definieren konnten, der als Qualitätsziel nicht unterschritten werden sollte, haben wir zusätzlich die Forderung der SVS überprüft, der zufolge eine offene Versorgung eines AAA nur in Krankenhäusern mit einer Klinikletalität $\leq 5 \%$ durchgeführt werden sollte [3]. Wir fanden 30 Kliniken mit einer Klinikletalität von im Mittel 7,2\% (5,0-10,0\%), die das Qualitätsziel der SVS nicht erreichten. Der MTL30 machte in dieser Kohorte $17,6 \%$ aus, sodass wir zunächst einmal, solange keine weiteren Untersuchungen vorliegen, lediglich einen MTL30 von unter $<17 \%$ als Qualitätsziel für unser Register bei OAR des AAA nennen können, ein Wert, den allerdings im Durchschnitt sämtliche Volumenquintile erreichten.

Einschränkend muss angemerkt werden, dass große Zentren in dieser Erhebung unterrepräsentiert waren, mit nur 12 von 37 Universitätskliniken. Durchschnittlich wurden jährlich von ca. 130 Krankenhäusern Daten erfasst, obwohl in Deutschland in ca. 500 Krankenhäusern AAA versorgt werden [10]. Ein Selektionsbias ist bei den Registerangaben demnach nicht auszuschließen, die Daten geben nur einen Ausschnitt aus der Versorgungsrealität wieder. Eine externe Validierung der Daten und ein Datenmonitoring waren technisch nicht möglich. Ob, wie aufgefordert, sämtliche Patienten einer Klinikgemeldet wurden, konnte nicht überprüft werden. Wenn demnach in dieser Arbeit vom Fallvolumen gesprochen wird, ist immer das Dokumentationsvolumen gemeint. Auch fragt es sich, ob der MTL30 bei den geringen Unterschieden in den Klinikquintilen als Qua- litätsziel tatsächlich der geeignetste Parameter ist (•Tab. 3 und 4). In zukünftigen Untersuchungen unseres Registers sollte deshalb vielleicht dem Vorschlag von Hardt et al. [7] gefolgt und der MTL22 als Qualitätsparameter untersucht werden, der Mortalität, Transfer und Liegezeit bereits nach 22 Tagen erfasst. Dieser Parameter war in der Analyse der kolorektalen Karzinomchirurgie dem MTL30 hinsichtlich der Sensitivität bei der Erfassung der postoperativen Morbidität mit $65 \%$ vs. $40 \%$ eindeutig überlegen. Unabhängig davon liegen aber bisher generell $\mathrm{zu}$ wenige Registererhebungen vor, die den MTL30 bewertet haben. Dieser Beitrag versteht sich als Ansatz, diese Lücke zu schließen.

\section{Schlussfolgerung}

Eine eindeutige Beziehung zwischen Krankenhausfallaufkommen und Klinikletalität ließ sich im AAA-Register des DIGG nicht aufzeigen. Das gleiche galt für den MTL30, der sich nicht zu dem Fallaufkommen einer Klinik korrelieren ließ. Ob demnach der MTL30 gegenüber der Erfassung von Klinikletalität und stationärer Liegezeit als Qualitätsparameter einen Zusatznutzen bietet, muss offenbleiben.

\section{Korrespondenzadresse}

\section{Y. Carmen Ahmadzadeh}

Klinik für Gefäß- und Endovascularchirurgie und des Universitären Wundzentrums, Klinikum der Goethe-Universität Frankfurt/M, Deutschland

Yasmin-Carmen.Ahmadzadeh@kgu.de

Univ.-Prof. Dr. med. Th. Schmitz-Rixen

Klinik für Gefäß- und Endovascularchirurgie und des Universitären Wundzentrums, Klinikum der Goethe-Universität Frankfurt/M, Deutschland

Schmitz-Rixen@em.uni-frankfurt.de

\section{Univ.-Prof. Dr. med. D. Böckler}

Klinik für Gefäßchirurgie und Endovaskuläre Chirurgie, Universitätsklinikum Heidelberg Heidelberg, Deutschland

dittmar.boeckler@med.uni-heidelberg.de 
Prof. Dr. med. R. T. Grundmann

Deutsches Institut für Gefäßmedizinische Gesundheitsforschung (DIGG) der Deutschen Gesellschaft für Gefäßchirurgie und Gefäßmedizin Berlin, Deutschland grundmann@medsachverstand.de

Funding. Open Access funding enabled and organized by Projekt DEAL.

\section{Einhaltung ethischer Richtlinien}

Interessenkonflikt. Y.C. Ahmadzadeh, T. SchmitzRixen, D. Böckler und R.T. Grundmann geben an, dass kein Interessenkonflikt besteht.

Für diesen Beitrag wurden von den Autoren keine Studien an Menschen oder Tieren durchgeführt. Für die aufgeführten Studien gelten die jeweils dort angegebenen ethischen Richtlinien.

Open Access. Dieser Artikel wird unter der Creative Commons Namensnennung 4.0 International Lizenz veröffentlicht, welche die Nutzung, Vervielfältigung, Bearbeitung, Verbreitung und Wiedergabe in jeglichem Medium und Format erlaubt, sofern Sie den/die ursprünglichen Autor(en) und die Quelle ordnungsgemäß nennen, einen Link zur Creative Commons Lizenz beifügen und angeben, ob Änderungen vorgenommen wurden.

Die in diesem Artikel enthaltenen Bilder und sonstiges Drittmaterial unterliegen ebenfalls der genannten Creative Commons Lizenz, sofern sich aus der Abbildungslegende nichts anderes ergibt. Sofern das betreffende Material nicht unter der genannten Creative Commons Lizenz steht und die betreffende Handlung nicht nach gesetzlichen Vorschriften erlaubt ist, ist für die oben aufgeführten Weiterverwendungen des $\mathrm{Ma}$ terials die Einwilligung des jeweiligen Rechteinhabers einzuholen.

Weitere Details zur Lizenz entnehmen Sie bitte der Lizenzinformation aufhttp://creativecommons.org/ licenses/by/4.0/deed.de.

\section{Literatur}

1. Antoniou GA, Antoniou SA, Torella F (2020) Editor's choice-endovascular vs. Open repair for abdominal aortic aneurysm: systematic review and meta-analysis of updated peri-operative and long term data of randomised controlled trials. Eur JVasc Endovasc Surg 59:385-397

2. Bulder RMA, Bastiaannet E, Hamming JF, Lindeman JHN (2019) Meta-analysis of long-term survival after elective endovascular or open repair of abdominal aortic aneurysm. Br J Surg 106:523-533

3. ChaikofEL, Dalman R, Eskandari MKetal (2018) The Society for Vascular Surgery practice guidelines on the care of patients with an abdominal aortic aneurysm. J Vasc Surg 67:2-77

4. Dua A, Furlough CL, Ray Het al (2014) The effect of hospital factors on mortality rates after abdominal aortic aneurysm repair. JVasc Surg 60:1446-1451

5. Grundmann RT, Thomsen J (2020) Bauchaortenaneurysma. In: Debus ES, Grundmann RT (Hrsg)
Versorgungsqualität in der operativen Medizin. Springer, Deutschland

6. Hardt J, Buhr HJ, Klinger C et al (2018) Qualitätsindikatoren für die onkologische Kolonchirurgie: Evidenzbasierte Entwicklung eines Indikatorensets für die Ergebnisqualität. Chirurg 89:17-25

7. Hardt J, Doyon F, Klinger C et al (2019) MTL, a composite measure for objectively profiling hospitals on surgical performance in colorectal cancer surgery. Int J Colorectal Dis 34:889-898

8. Nimptsch U, Mansky T (2017) Hospital volume and mortality for 25 types of inpatient treatment in German hospitals: observational study using complete national data from 2009 to 2014. BM Open 7(9):e16184

9. Schmitz-Rixen T, Steffen M, Böckler D, Grundmann RT (2020) Versorgung des abdominellen Aortenaneurysmas (AAA) 2018. Register-Bericht des DIGG der DGG. Gefässchirurgie 25:117-123

10. Trenner M, Kuehnl A, Salvermoser M et al (2018) Editor's choice - high annual hospital volume is associated with decreased in hospital mortality and complication rates following treatment of abdominal aortic aneurysms: secondary data analysis of the nationwide German DRG statistics from 2005 to 2013. Eur J Vasc Endovasc Surg 55:185-194

11. Wanhainen A, Verzini F, Van Herzeele I et al (2019) Editor's choice-European Society for Vascular Surgery (ESVS) 2019 clinical practice guidelines on the management of abdominal aorto-iliac artery aneurysms. Eur JVasc Endovasc Surg 57:8-93

12. Wellner UF, Grützmann R, Keck T et al (2018) Qualitätsindikatoren für die Pankreaschirurgie: Wissenschaftliche Herleitung und klinische Relevanz. Chirurg 89:32-39

13. Wiegering A, Wellner U, Seyfried F et al (2017) MTL30 als Surrogatparameter der Behandlungsqualität chirurgischer Erkrankungen: Etablierung anhand der StuDoQ-Register der Deutschen Gesellschaft für Allgemein- und Viszeralchirurgie. Chirurg 88:977-982

14. Wiegering $A$, Buhr $\mathrm{HJ}$, Klinger $C$ et al (2018) Qualitätsindikatoren für die Chirurgie des Rektumkarzinoms:EvidenzbasierteEntwicklungeines Indikatorensets für die Ergebnisqualität. Chirurg 89:26-31

15. Yin $\mathrm{K}$, Locham SS, Schermerhorn ML, Malas MB (2019) Trends of 30-day mortality and morbidities in endovascular repair of intact abdominal aortic aneurysm during the last decade. J Vasc Surg 69:64-73

16. Zettervall SL, Schermerhorn ML, Soden PA et al (2017) The effect of surgeon and hospital volume on mortality after open and endovascular repair of abdominal aortic aneurysms. J Vasc Surg 65:626-634

17. Zettervall SL, Soden PA, Buck DB et al (2017) Significant regional variation exists in morbidity and mortality after repair of abdominal aortic aneurysm. JVasc Surg 65:1305-1312
Hier steht eine Anzeige. Springer 
Hier steht eine Anzeige.

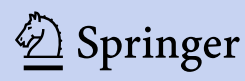

